\title{
Haptics and Graphic Analogies for the Understanding of Atomic Force Microscopy
}

\author{
Guillaume Millet $^{\mathrm{a}}$, Anatole Lécuyer ${ }^{\mathrm{b}}$, Jean-Marie Burkhardt ${ }^{\mathrm{c}}$, Sinan Haliyo ${ }^{\mathrm{a}}$, \\ Stéphane Régnier, ${ }^{\mathrm{a}, *}$ \\ ${ }^{a}$ UPMC Univ Paris 06, UMR 7222, ISIR, F-75005, Paris, France \\ ${ }^{b}$ INRIA/IRISA, Campus universitaire de Beaulieu, F-35042, Rennes CEDEX, France \\ ${ }^{c}$ IFSTTAR, LPC, F-78000 Versailles, France
}

\begin{abstract}
This paper aims to evaluate the benefits of using virtual reality and force-feedback to help teaching nanoscale applications. We propose a teaching aid that combines graphic analogies and haptics intended to improve the grasp of non-intuitive nanoscale phenomena for people without prior knowledge of nanophysics. We look specifically at the most important nanophysical phenomenon, namely, the behavior of the probe of an Atomic Force Microscope (AFM) as it approaches a sample. The results from experiments carried out with 45 students indicate that a "magnet-spring" analogy helped beginners to establish the link between the behavior of a probe and its force-distance curve. The addition of haptic feedback increased focus about forces and improved the interpretation of the effect of cantilever stiffness. Haptic feedback and the analogical representation were very much appreciated by the subjects and had an impact on the construction of a mental model. Taken together, our results show a positive influence of using haptic feedback and graphic analogies, especially when students are first exposed to the notions that are in effect at the nanoscale.
\end{abstract}

Keywords: Haptic I/O, analogies, education, micro/nano technology, human factors

\section{Introduction}

Recent advances in the field of nanotechnology, which result from the development of new imaging and manipulation instruments, led to the discovery of intangible phenomena at the nanoscale. These phenomena are difficult to comprehend, especially because they require to make "a mental jump" across spatial scales in a different "world" (Tretter et al., 2006b). The main tool for imaging, measuring, and manipulating matter at this scale is the atomic force microscope, or AFM. It uses a micro-scale cantilever with

\footnotetext{
${ }^{*}$ Corresponding auhtor. Tel.: +331442728 79; fax: +33144275145.

Email addresses: guillaume.millet@isir.upmc.fr (Guillaume Millet), anatole. lecuyer@irisa.fr (Anatole Lécuyer), jean-marie.burkhardt@ifsttar.fr (Jean-Marie Burkhardt), sinan.haliyo@isir.upmc.fr (Sinan Haliyo), stephane.regnier@isir . upmc.fr (Stéphane Régnier)
} 
a sharp tip (probe). The deflection of the cantilever is measured with sub-micrometer precision making it possible to calculate the forces at play between the probe and a sample, provided that the cantilever stiffness is known. Initially developed solely as a measuring device, the AFM is also capable of manipulating nano-objects. However, the use of this technique is limited by a lack of useful visual feedback, inherent to scanning probe imaging, and by scaling effects of nanoscale physics.

Virtual reality (VR) techniques and haptic feedback have been developed to enhance the operator's experience of the phenomena at play at the nanoscale. Specifically, vR can provide users with a reconstructed $3 \mathrm{D}$ view of a previously scanned manipulation scene, which can be displayed at human scale and from different vantage points. Using a haptic device, users can benefit from force feedback, within the limits of the system's measurement capabilities. Several "telenanorobotic" systems have been developed along these lines over the past decade, combining an AFM with a haptic device and an augmented reality human-machine interface (Vogl et al., 2006; Li et al., 2005). It must be noted, however, that efficient "telenanomanipulation" is a technical challenge (Xie and Régnier, 2012; Sitti, 2007). Using a force-feedback device to transcribe nanoscale forces is far from being straightforward because of adhesion effects (Israelachvili, 1992), large dynamics, small masses involved, and stability concerns in bilateral coupling (Bolopion et al., 2012). The reader is referred to (Ferreira and Mavroidis, 2006) for a review of the use of VR and haptics for nanorobotics.

Another important aspect of nanomanipulation is education and training. There is currently a new generation of engineers specializing in nanotechnology whose training involves becoming familiar with nanoscale intricacies (Roco, 2003). In addition, the introduction of nanosciences and nanotechnologies in secondary education should contribute to the acquisition of a "nanoscientific-literacy" (Hingant and Albe, 2010). Thus, nanosciences and nanotechnologies are an emerging field in science education which may undergo major expansion in the coming years (Hingant and Albe, 2010). As an emerging field, however, the evidence regarding the design of representations and educational strategies that could enhance students' learning and understanding of phenomena at the nanoscale is but little and somewhat conflicting; for recent reviews, see (Hingant and Albe, 2010; Minogue and Jones, 2006).

An interactive VR tool may contribute to promoting awareness and learning of the different aspects of nanoscale phenomena by providing a dynamic and more accurate direct experience of these phenomena (Tretter et al., 2006a; Park et al., 2010) through both visual and haptic feedback. Moreover, as the use of analogies has been shown as helpful for learning in physics (Podolefsky and Finkelstein, 2006), we were interested in evaluating the benefit of using macro-scale notions for the understanding of more exotic nanoscale phenomena.

We propose in this paper to evaluate the educational benefits of using an AFM simulation interfaced to a haptic device. This work extends the description and analysis of the experiment originally conducted and described in (Millet et al., 2008). Since it is difficult and expensive to set up a simple and repeatable nanomanipulation experiment for user evaluation, we built a vR-based simulator coupled to a haptic device. The simulator reproduces the behavior of an AFM probe during an "approach-retract" (AR) procedure. It is based on our previous work on micro- and nanomanipulation that provides us with new theoretical insights and experimental data (Haliyo et al., 2003). The 
AR procedure involves dealing with the adhesion forces between the probe and a substrate. At the nanoscale, the phenomenon resembles macro-scale magnetic attraction whereby the behavior of the AFM probe is similar to that of a magnet attached to a spring approaching a ferromagnetic surface. We have therefore implemented this analogy as a second graphic representation in VR.

The following section presents an overview of previous work with pedagogical concerns in nanoscience using VR. After, the paper describes the experimental set-up and method to evaluate the benefit of using force feedback and the graphic analogy. Then, the results regarding participants' performance and preferences are presented and discussed.

\section{Related work}

\section{Mental models, human-computer interaction, and learning}

Mental models are internal mental representations that reflect the "world" situation as described or depicted in external representations like text, animations, etc. There is a large literature on mental models in several fields of psychology from human-computer interaction (HCI) to text comprehension, problem solving, learning and education. In HCI, mental models provide an adequate framework for addressing the issues related to users' understanding of systems they used. In the other domains, research on mental models in learning has provided theories to explain processes and difficulties related to acquiring concepts and building accurate representations of situations depicted in texts and other external representations. This approach has been found as particularly adapted for scientific learning (e.g., Tretter et al., 2006a).

In this perspective, it is assumed that mental models of situations are initially built up from processing one or several external representations and make extensive use of the subject's existing domain knowledge. They are produced by inferences and are also a source for making new inferences (Johnson-Laird, 1983; Kintsch, 1988). They are consequently not bound to specific sensory channels and are more abstract than perceptual images (Schnotz and Bannert, 2003) since both irrelevant details from perceptual images are omitted and prior knowledge can be inversely included. Constructing mental models of systems entails understanding structural causal interactions as well as functional relationships among entities in the situation to be understood. Specifically, structural causal interactions reveal how one entity is causally related to another (i.e., similar to causal chains in events or stories); functional relationships describe how a change in one parameter leads to a change in another parameter or the entire system (i.e., similar to a math function).

Another characteristic is that the building of a mental model is highly constrained by the limited capacity of working memory (Baddeley, 1992; Sweller et al., 1998). Because learning depends on what and how information is actually processed in working memory, three sources of cognitive load (intrinsic, extraneous, and germane) can result in overload. Intrinsic cognitive load is connected with the nature of the material to be learned, its novelty and complexity, the availability of relevant a priori knowledge in long term memory, etc. Extraneous cognitive load is related to the designed instructional materials provided to the learner. This source of cognitive load does not 
contribute to learning since it reduces working memory capacity for learning. Germane cognitive load is related to the resources of free working memory actually involved for deeper construction and automation of schemata. This source depends, on the one hand on the resources left free in working memory by the intrinsic and the extraneous cognitive load and, on the other hand, on the engagement and motivation of the learner to achieve the learning task. From an educational viewpoint, the main idea is to eliminate extraneous cognitive load by an adequate design of external representations and to increase germane cognitive load within the constraints of working memory and the intrinsic load. In the specific context of interactive multimedia learning environment, several principles has been empirically demonstrated to reduce cognitive load (e.g., Mayer, 2001; Bannert, 2002).

Research on mental models has investigated how they are built from various types of external representations. A common approach has been to emphasize the role of the sensory channel (e.g., auditory or visual) or the presentation modality (e.g., text or picture) on learning effectiveness (e.g., Mayer, 2001; Sweller et al., 1998). In this line, numerous works have been carried out on the effectiveness of animations to convey information about change in time and change over time (Chan and Black, 2006b; Tversky et al., 2002; for a review, see Bétrancourt and Tversky, 2000). More recently, there have been conflicting evidence that haptics can enhance learning by providing a support to building mental models (Minogue and Jones, 2006; Chan and Black, 2006a,b; Park et al., 2010; Young et al., 2011).

\section{Benefits of haptics to understanding, learning, and education}

Research on haptics (for a more complete presentation, see Lederman and Klatzky, 2009 for example) has initially concerned the psychophysics of tactile and kinesthetic perception. Haptic perception and representation are based on inputs from mechanoreceptors and thermoreceptor subsystems in the skin, as well as on position-sensing and force-sensing mechanisms that exist in muscles, tendons and joints (Lederman et al., 1996). By exploiting the haptic system, subjects are able to extract different kinds of information from the manual exploration of objects (Lederman and Klatzky, 1987) such as their material properties (e.g., surface texture, hardness, thermal, weight), their geometric properties (those pertaining to shape and size), their mechanical properties (e.g., motion of an object part), and their functional properties (as determined from object structure). Part of the research has concentrated on the specificity of haptics as compared to other sensory modalities, mainly vision (e.g., Gaißert et al., 2010) whereas other works have addressed issues related to the combination of haptics with other sensory modalities in perception and representations (e.g., Hecht et al., 2008a).

These previous works have clarified capabilities and limitations associated with the haptic system. For example, geometric tasks that require haptic recognition without vision are performed relatively poorly, whereas material properties are processed more precisely and quickly. Furthermore, there is dominance of vision over haptics on extracting geometric information because vision can extract spatial details far more precisely and faster than can any haptic exploration procedure (Lederman et al., 1996).

At the same time, there have been a growing number of studies on haptics devices and human-computer interaction, as well as on illusions generated by combining haptics with vision or auditory stimuli. Recent work by Lederman and Klatzky (2004) has 
interestingly investigated the effect of various constraints on haptics perception and representation. They have shown in a series of experiments that, depending on the nature of constraints on manual exploration, the available cutaneous or kinesthetic information is reduced. For example, if the task requires the recognition of objects, the performance becomes poorer when the haptic field of view is reduced (e.g., exploring with one finger instead of five). More relevant to our study, they also demonstrated that a rigid link like a probe or current haptic devices with a single point contact interaction limits the normally rich cutaneous information to highly localized vibrations and the kinesthetic information to kinematic and dynamic cues produced by moving the rigid link. Such a reduction can have an instructional benefit in our context, by reducing the amount of haptic information to process and by orienting the exploration towards information required to achieve the learning task. Most of these works however have considered elementary tasks often with non-semantic stimuli, which leaves rather unexplored the issues related to the contribution of haptics to learning in complex domains.

Interactive haptics provides a two-way communication channel between the learner and the system which enables the learner to fully manipulate the to-be-learned materials through their hand control and, thus, to actively explore it while reasoning about the presented material. The addition of haptic feedback in vR could have an effect on the learner's performance and workload by increasing the amount of information to be processed (e.g., Moreno and Mayer, 1999).

\section{Benefits of analogical representations to understanding, learning, and education}

Graphical animation representing dynamics has been shown as particularly interesting to depict phenomena with multiple spatial or temporal evolutions (Bétrancourt and Tversky, 2000). When used in an interactive manner, dynamical representations can also prompt subjects to be more active in the learning process (Hegarty, 2004). Depending on how it is designed, external representations can help learners and users by facilitating the acquisition and process of meaningful information as well as its transfer to new situations (Sweller, 1994; Mayer, 2001). However, external representations can be difficult to follow because they require the learner to understand how the representation encodes information and identify its relevant aspects amongst (potentially numerous) available features.

Another major topic in science education concerns the use of analogies in teaching and understanding science (Duit et al., 2001; Niebert et al., 2012). It has been shown that analogical representations can help students building and reflecting upon mental models based on their prior knowledge (Gentner, 1989). Reasoning by analogy requires to map a familiar structure (a set of properties) of the source already known with the new structure of the target. It has been recognized as a central process for learning conceptual changes in several theories, be it spontaneous with multiple analogies as in physics reasoning (e.g., Clement, 1988) or be it induced by instructional materials (e.g., Trey and Khan, 2008). However, analogies can also mislead learner's thinking and learning processes (e.g., Duit et al., 2001).

Podolefsky and Finkelstein (2006) suggested that graphical representations (e.g., graphs) play a key role in the use of analogy by cuing students to focus on particular 
characteristics of physical phenomena. They showed that analogies can promote learning in large-scale introductory physics courses. They suggested that instructors attend to the myriad ways that analogical representations can be interpreted and seek to create blends between productive representations.

Beyond the richness and multimodal properties of using a VR-based simulator for AFM manipulation, our situation is also grounded on constructivist and "learning by doing" theories in that learners have both to concretely experiment, formulate hypotheses, and test them through the manipulation of the simulator. Briefly, the main idea shared by these theories is that learning fundamentally results from the subject's activity and that knowledge is built in the course of unceasing interactions with surrounding objects and culture. Learning is thus not the mere effect of being exposed to and being given the task of understanding some educational material, but depends on the interactions between the subjects and their environment in the context of problem solving or exploratory activities. These theories have been developed in the frame of various objects of interest and research approaches, for example cognitive development (Piaget, 1964; Vygotsky, 1978), educational interactions (Bruner, 1966) and educational technologies (Papert, 1980; for an application to virtual environments for learning, see Winn, 2003; Burkhardt et al., 2003).

\section{Nanoscience education}

A growing research community is studying the effectiveness of using VR simulations for the enhancement of students' understanding of complex scientific topics. Providing force feedback to students with a low-cost "haptic paddle", Okamura et al. (2002) suggested that educational haptics is appropriate for teaching notions in dynamic systems. An evaluation of hands-on sessions in (Grow et al., 2007) supports this idea but is limited by the lack of a control group.

Reiner (2008) showed that touch is an efficient additional channel for providing details during visualization and suggested that learning environments would benefit from using both visual and haptic information to support visualization. This suggestion was based on two previous studies. The first used force feedback for protein-ligand docking in biomolecular chemistry (Brooks, Jr. et al., 1990). The second employed tactile feedback for the visualization of force fields (Reiner, 1999). According to the thorough review by Minogue and Jones (2006) on haptics in education, these studies tend to be somewhat inconclusive in providing evidence that the addition of haptics may enhance the learning process. Although there is no doubt that the heightened sense of immersion provided by haptics makes the learning process more engaging, Minogue and Jones (2006) concluded that "it is still largely unknown whether augmenting instruction with the sense of touch can exploit experiential, embodied, and tactile knowledge that might not otherwise be called upon by students" (p. 342).

In the context of nanoscience education, some recent work was concerned with the impact of VR, mainly with haptic augmentation. Table 1 summarizes the content of

these studies. For teaching biomolecular chemistry, Persson et al. (2007) evaluated a haptic system in which students were able to manipulate a ligand and feel its interactions in the docking process. They found no obvious advantage for learning from the addition of force feedback to their system. Nevertheless, the students' answers showed 
Table 1: Descriptive summary of related studies in nanoscience education

\begin{tabular}{|c|c|c|c|c|c|}
\hline Study & Participants & Device & Stimuli & Measures & Dependent variable(s) \\
\hline $\begin{array}{l}\text { Persson et al. } \\
(2007)\end{array}$ & $\begin{array}{l}23 \text { Undergraduate } \\
\text { students }\end{array}$ & 6D Рhantom & $\begin{array}{l}\text { Molecular docking } \\
\text { simulation }\end{array}$ & $\begin{array}{l}\text { Knowledge tests, opinion } \\
\text { questionnaire, docking tasks }\end{array}$ & $\begin{array}{l}\text { Understanding of docking } \\
\text { interactions }\end{array}$ \\
\hline $\begin{array}{l}\text { Jones et al. } \\
(2003)\end{array}$ & $\begin{array}{l}43 \text { High school } \\
\text { students }\end{array}$ & 6D Phantom & $\begin{array}{l}\text { Teleoperation of an AFM } \\
\text { probe touching a virus }\end{array}$ & $\begin{array}{l}\text { Knowledge tests, opinion } \\
\text { questionnaire, clay modeling, interview }\end{array}$ & $\begin{array}{l}\text { Understanding of viruses } \\
\text { and of AFM imaging process }\end{array}$ \\
\hline $\begin{array}{l}\text { Jones et al. } \\
(2006)\end{array}$ & $\begin{array}{l}36 \text { High school } \\
\text { students }\end{array}$ & $\begin{array}{l}\text { mouse, } \\
\text { 2D joystick, } \\
\text { 3D PHANTOM }\end{array}$ & $\begin{array}{l}\text { Simulation of an AFM } \\
\text { probe touching a virus }\end{array}$ & Knowledge tests, opinion questionnaire & $\begin{array}{l}\text { Understanding of physical } \\
\text { properties of viruses }\end{array}$ \\
\hline $\begin{array}{l}\text { Minogue et al. } \\
(2006)\end{array}$ & $\begin{array}{l}80 \text { Middle school } \\
\text { students }\end{array}$ & 6D Phantom & $\begin{array}{l}\text { Simulation of an animal } \\
\text { cell }\end{array}$ & Knowledge tests, opinion questionnaire & $\begin{array}{l}\text { Understanding of the } \\
\text { structure and function of an } \\
\text { animal cell }\end{array}$ \\
\hline $\begin{array}{l}\text { Marchi et al. } \\
(2005)\end{array}$ & $\begin{array}{l}60 \text { Undergraduate } \\
\text { students }\end{array}$ & 1D ERGos & $\begin{array}{l}\text { AR cycles of real and } \\
\text { virtual AFM probes }\end{array}$ & $\begin{array}{l}\text { Knowledge tests, curve drawing, } \\
\text { opinion questionnaire }\end{array}$ & $\begin{array}{l}\text { Understanding of AFM and } \\
\text { AR cycle }\end{array}$ \\
\hline
\end{tabular}

that force feedback sharpened the students' understanding of the forces involved in the docking process.

In biology, Jones et al. (2003) used a vR platform called nanoManipulator with a Рналтом haptic device connected to an AFM to investigate the impact of its use on students' understanding of virus morphology and of the AFM imaging process. This study found that students developed more accurate conceptions of virus morphology, moving from a $2 \mathrm{D}$ to a $3 \mathrm{D}$ understanding of viruses, but no difference was detected as a result of the use of haptics. A second experiment assessed the addition of different types of haptic feedback (a 6D PнAnтom device and a 2D joystick) (Jones et al., 2006). They found a correlation between the sensitivity of the haptic device used and the number of haptic terms and of spontaneous analogies that students used to describe the virus. A third study on the understanding of the structure of an animal cell mainly showed a motivational effect of using haptics (Minogue et al., 2006).

In scanning microscopy, Marchi et al. (2005) developed a multi-sensory (visual, auditory and haptic) platform equipped with a real-time physics engine. It was used in the teaching of the one-dimensional AR nanophysical phenomenon to master's level students. They report that the students provided a better description of the phenomenon compared to a session where a conventional AFM description was used. This result may be due to the possibility in VR simulations to modify different parameters from one extreme to the other, facilitating the observation of their influence. This explanation agrees with the conclusion offered by Finkelstein et al. (2005) who suggested that properly designed computer simulations are useful tools for a variety of contexts that can promote student learning in the appropriate contexts. Yet, the experimental plan did not directly compare the benefit of using haptic feedback during the student understanding process.

In addition to the traditional AFM visual representation showing a top view of the cantilever, other representations can aid in understanding. For instance, the AR interaction described in (Marchi et al., 2005) used an atomic representation, consisting of a triangular tip interacting with an elastic layer of atoms depicting the sample surface, 
and a vertical line with zero free length symbolizing the cantilever. Another representation aims to help the understanding of the energetic behavior of the probe by using the analogy of a ball rolling down a potential landscape (Marlière et al., 2004). As pointed out by Podolefsky and Finkelstein (2006), analogies can be used to promote students' understanding and learning in physics. However, in the context of AFM education, there has not yet been a study of the use of possible graphic analogies.

\section{This work}

Our study focuses on the approach-retract (AR) phenomenon because it is the most significant phenomenon during nanomanipulation. In this work, we aim to explore the benefit of using a haptic device and an analogous graphic representation of the AR phenomenon on subjects' performance relatively to their understanding of the phenomenon. Does providing haptic feedback result in a better understanding compared to providing only graphic representation? Does providing an appropriate analogical representation enhance the understanding compared to providing the realistic one, in particular for novices?

We carried out an experiment with four group conditions, with or without haptics and with realistic or with analogical representation, where novice subjects were asked to experience the mechanical behavior of the virtual AFM probe when it touches a sample surface. Preliminary analyses presented in (Millet et al., 2008) showed a positive effect of both haptics and graphic analogy. Here we extend these results and analyze subjects' understanding both in terms of speed and of accuracy using various analytical techniques related to different types of measures of learning: spontaneous verbal reports, drawings, and curve identifications.

\section{Experimental set-up}

The experimental set-up made it possible for a user to manipulate a virtual AFM probe. The simulation provided force feedback and two alternative graphic representations.

\subsection{Approach-retract phenomenon}

This phenomenon accounts for the mechanical behavior of the AFM probe when it touches a sample surface in the normal direction. A typical measurement is to record the force applied by the substrate on the tip versus the distance between the substrate and the cantilever base. The profile of a force-distance curve of a silicon tip touching a polystyrene surface in ambient conditions is exemplified in Fig. 1. Examples of experimental AR curves in ambient conditions can be found in (Gauthier et al., 2005).

Far away from the substrate, forces acting on the probe at distance are negligible, so the cantilever is undeformed (point $\mathbf{A}$ ). Approaching point $\mathbf{B}$, attraction forces rapidly increase and bend the cantilever. During the approach phase, bending is much smaller than that during the retraction phase (by more than one hundred times in ambient conditions) and thus, is generally not visible at the scale of the whole curve, unless very sensitive probes are used. When point $\mathbf{B}$ is reached, an instability (positive gradient) appears because the attraction force gradient overcomes the cantilever stiffness. The 


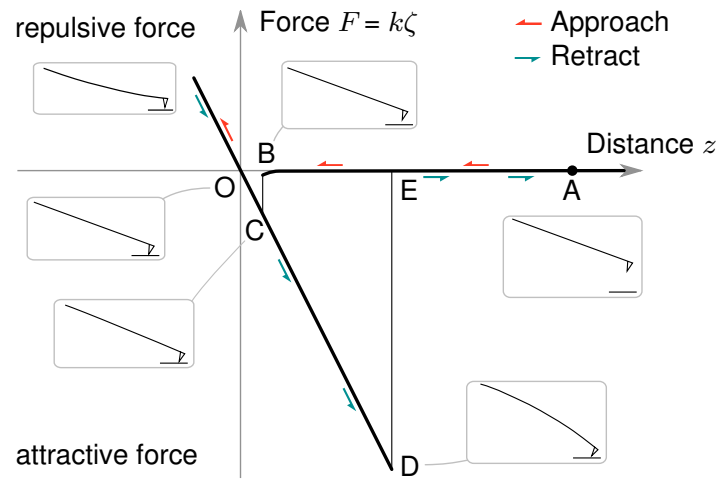

Figure 1: Force-distance curve of an AR phenomenon. It shows the evolution of the force applied by the substrate on the tip versus the distance between the substrate and the cantilever base. See text for explanation.

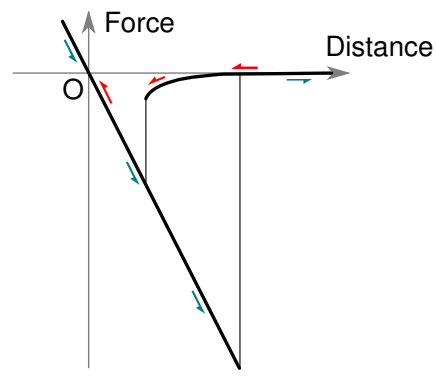

Figure 2: AR force-distance curve with an electrostatic force.

tip curtly snaps on the substrate; this snap-in event corresponds to the segment $\mathbf{B C}$ on the curve.

From point $\mathbf{C}$, the tip is repelled by contact forces depending on the contact mechanics. During retraction, the tip remains stuck to the substrate and the cantilever bends until the cantilever stores sufficient elastic energy during bending to overcome adhesion. Point $\mathbf{D}$ represents an unstable equilibrium point between the adhesion and the elastic force of the cantilever. This threshold point is commonly called the pulloff. Then, once the equilibrium is broken, a second fast force variation of the AR curve occurs, snapping out the tip to its rest position (point $\mathbf{E}$ ).

To sum up, forces of attraction and adhesion yield a hysteretic behavior with two thresholds that depend on several parameters such as cantilever stiffness, contact conditions (material, surface rugosity and oxidation, contact duration, etc.) and environmental conditions (presence of electric charges, humidity, pressure, temperature). We focused our experiment on two of them: cantilever stiffness and electric charges. The latter create electrostatic forces, which could be neglected for non-charged and grounded surfaces, but for a non-conducting substrate, charges can be generated during the contact interaction and result in increased electrostatic forces. Its longer range results in an earlier snap-in and a higher cantilever deflection before the snap-in (see Fig. 2).

In ambient conditions, attraction forces occurring before the tip makes contact with 


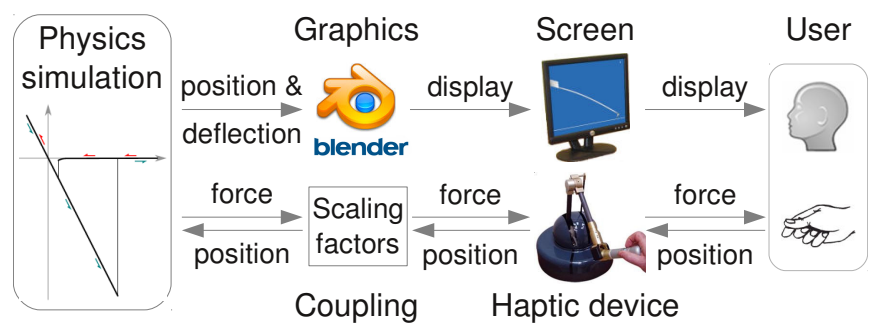

Figure 3: Haptic and virtual reality system overview.

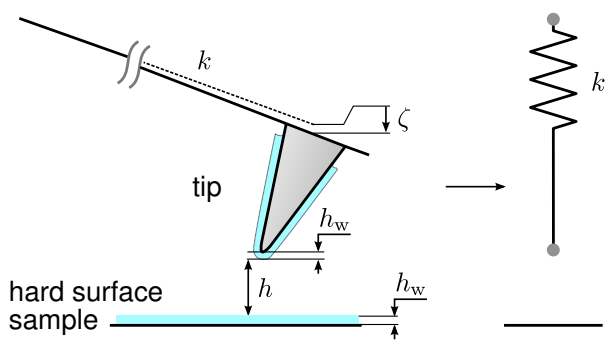

Figure 4: Spring model of the contact mechanics.

the substrate are mainly due to van der Waals and electrostatic forces. Moreover, ambient conditions involve the presence of a thin layer of water on surfaces, which adds capillary forces and tends to increase the pull-off threshold.

\subsection{Haptic and virtual reality system}

The experimental system, illustrated in Fig. 3, comprises a real-time simulation of the AR phenomenon, along with a 3D computer graphics interface and a force-feedback device. The simulation computes the bending of the cantilever, according to its position. Bending can be visualized or felt by the user as force feedback.

\subsubsection{Physics simulation}

The AR simulation used in (Marlière et al., 2004) employed a dynamic simulation engine. The mechanical time constant of contact mode cantilevers, however, is typically shorter than $1 \mathrm{~ms}$. Therefore, at the time scale of human perception, the behavior of the probe during AR can be assumed to be quasi-static. As a result, we reduced the simulation to calculating the cantilever deflection at each position between the transient moments, the snap-in and the pull-off. These moments can be considered to be state transitions from contact and non-contact, and vice-versa.

The form factor of the cantilever results in a linear mechanical behavior within the domain of small deformations, so the probe is modeled by a spring without mass (see Fig. 4). The force $F$ applied by the substrate on the tip entails a cantilever deflection $\zeta$ such as $\zeta=F / k$ where $k$ is the cantilever stiffness. Two cases of simulation are considered depending on the type of non-contact forces acting during approach. 
The first case assumes that electrostatic forces can be neglected. During approach, the equilibrium equation, using the Derjaguin approximate expression of sphere/plane van der Waals forces, is written

$$
k \zeta_{\mathrm{a}}+\frac{H R}{6 h^{2}}=0,
$$

where $\zeta_{\mathrm{a}}$ is the deflection during approach, $h$ the distance between the tip and the substrate (between the water layers), $H$ the Hamaker constant, and $R$ the tip radius. The distance $z$ between the cantilever base position and the substrate is defined as $z=h-\zeta$. Equation (1) is a cubic polynomial and its solution obtained from Cardano's method is

$$
\begin{aligned}
\zeta_{\mathrm{a}} & =\frac{2 z}{3}\left(\cos \left(\frac{1}{3} \arccos \left(1-2 \frac{z_{\mathrm{si}}^{3}}{z^{3}}\right)\right)-1\right), z>z_{\mathrm{si}}, \\
z_{\mathrm{si}} & =\frac{3}{2}\left(\frac{H R}{3 k}\right)^{\frac{1}{3}},
\end{aligned}
$$

where $z_{\mathrm{si}}$ is the height of the snap-in threshold.

The second case considers electrostatic forces between the tip and the substrate. The simulation assumes that the electrostatic forces are much stronger than the van der Waals forces. During approach, the equilibrium equation, using the sphere-plane expression of the electrostatic force (Sausse-Lhernould et al., 2007), becomes

$$
k \zeta_{\mathrm{a}}+\pi \epsilon_{0} \frac{R^{2} V^{2}}{h(h+R)}=0,
$$

where $\epsilon_{0}$ is the vacuum permittivity and $V$ the electric potential difference. This equation is also a cubic polynomial and its solution can also be found with Cardano's method.

When $z$ reaches $z_{\mathrm{si}}$, the tip snaps on the substrate, which is assumed non-deformable. Hence, the deflection during retraction $\zeta_{\mathrm{r}}$ is proportional to $z, \zeta_{\mathrm{r}}=-z-2 h_{\mathrm{w}}$, where $h_{\mathrm{w}}$ is the water layer thickness. The simulation returns to the approach phase when the applied force reaches the pull-off force $F_{\mathrm{po}}$. The pull-off force is calculated according to the Maugis-Dugdale contact model (Carpick et al., 1999).

During the experiments, we used the numerical values related to a silicon tip interacting with a polymer sample surface through a thin water layer of $h_{\mathrm{w}}=8 \mathrm{~nm}$ on each surface. With a flexible cantilever of tip radius $R=10 \mathrm{~nm}$ and stiffness $k=0.2 \mathrm{~N} / \mathrm{m}$, the resulting thresholds in the first case were $F_{\mathrm{po}}=25 \mathrm{nN}$ and $F_{\mathrm{si}}=0.13 \mathrm{nN}$. The force just before the snap-in was roughly two hundred times weaker than $F_{\mathrm{po}}$. With linear scaling factors between the nanoscene and the haptic device, the friction of the haptic device masked this pre-snap-in attraction force, which, as a result, became imperceptible. In the second case, the electric potential difference was $25 \mathrm{~V}$. This increased the snap-in to $F_{\mathrm{si}}=2.3 \mathrm{nN}$, which was now perceptible with sufficient attention.

\subsubsection{Visual feedback}

Two virtual representations were tested as illustrated in Fig. 5, using the opensource software Blender ${ }^{1}$ for the rendering. The first shows the side view of a cantilever

${ }^{1}$ WWW. blender. org 


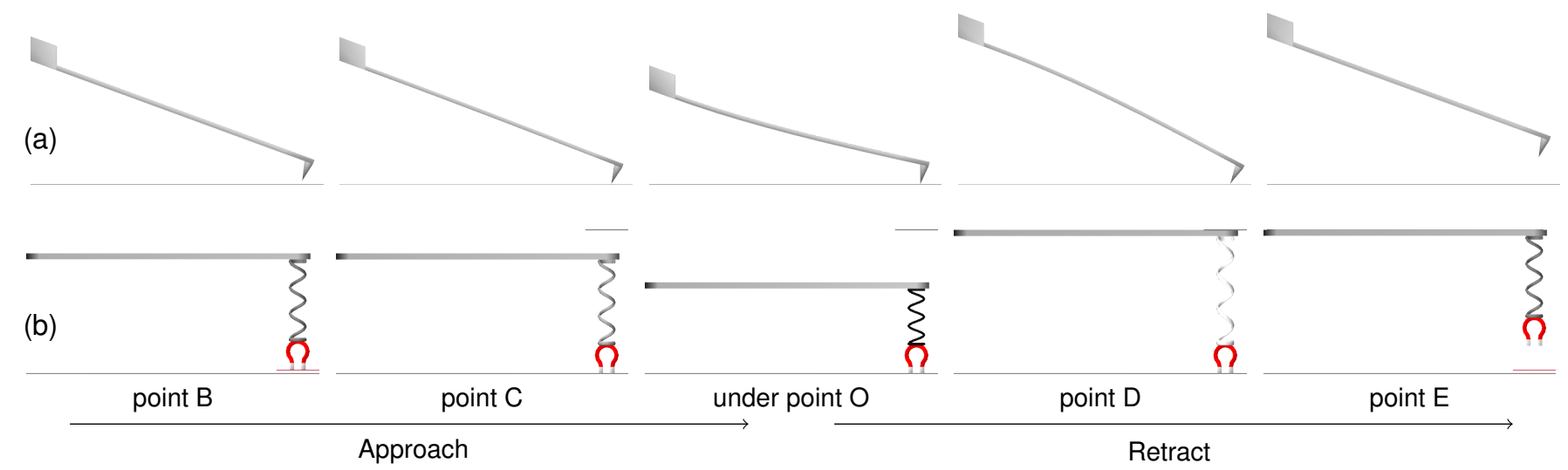

Figure 5: Virtual representations of (a) the cantilever and (b) its magnet-spring analogy, during approach and retract phases.

beam, which was shortened about $10 \times$ relatively to a real one in order to magnify its bending. Its mounting angle is 20 degrees, like in a conventional AFM, such that its base is clear from the substrate.

The second representation provides a behavioral analogy with graphical cues. There is no conventional analogy representing the behavior of an AFM probe. Planinšič and Kovač (2008) and Greczyło and Debowska (2006) have used a thin beam and magnetic forces to simulate attraction force in a human-scale teaching model of the AFM. Indeed, a good candidate for the analogy should provide a human-scale system combining elasticity and decreasing attraction force, resulting in a hysteretic behavior. Another potential analogy for elasticity was found in (Clement, 1988) where experts generated analogies with a cantilever beam to solve a problem with springs. After discussion with physics teachers, we decided to use a system made of a magnet attached to a well-damped spring touching a ferromagnetic surface. Students are likely more aware of the behavior of springs and magnetic forces than that of AFM probes, both from experience in everyday life and also from previous classes in physics. In particular, the magnet suggests the existence of a decreasing attraction force and of a snapping behavior when getting closer to the surface; on the other hand, the cantilever tip does not suggest any cue on the interaction with the surface.

In addition to the analogy, the second representation shows several graphical cues aiming at drawing attention to the influence of simulation parameters such as the type of attraction forces or the cantilever stiffness. Two visual marks symbolize and locate the snap-in and pull-off thresholds, in the vicinity of the observed area. The snap-in mark appears during approach and is located where the magnet snaps to contact. The pull-off transient, which is related to the base position of the cantilever, appears during retract and is placed above the horizontal bar, where the magnet takes off. Lastly, color variation of the spring provides an ecological and redundant information to the visual elongation to aid in its detection during approach, in particular before the snap-in. 


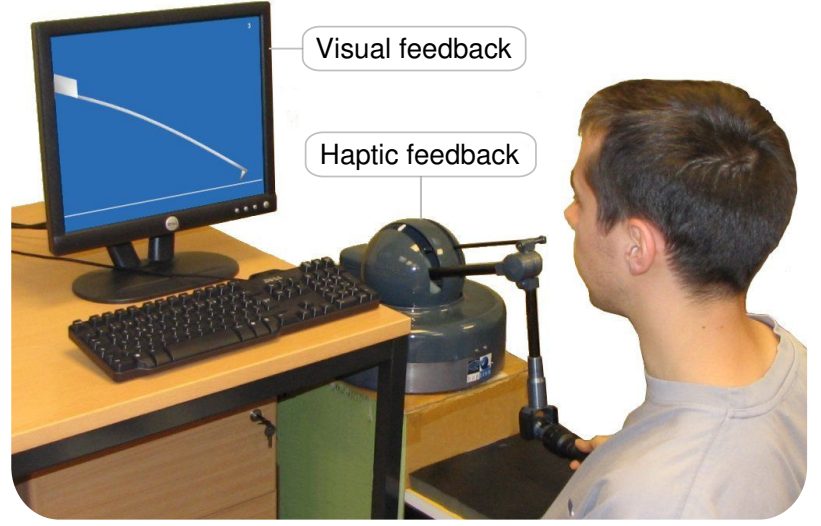

Figure 6: Overview of the experimental set-up.

\subsubsection{Force feedback}

The simulation and the haptic device are coupled in impedance mode: the device sends its vertical position to the simulation as the cantilever base position, and receives the vertical force to render. This force depends on the cantilever deflection and stiffness, as explained in Section 3.2.1. It is rendered to the haptic device with a constant scaling ratio. This ratio is determined by the rendered pull-off force, which was fixed at $7 \mathrm{~N}$.

The experimental set-up used a simulation written in $\mathrm{C}$ on a $3.2 \mathrm{GHz}$ Pentium 4 PC coupled to a VIRTUOSE 3D10-20 haptic device from Haption s.A. Haptic control was updated at a rate of $1 \mathrm{kHz}$.

\section{Experiment}

The aim of the study was to explore the effects of using haptics and graphic analogies on the students' understanding of the AR phenomenon. For this purpose, we carried out an experiment with four group conditions, with or without haptics and with realistic or with analogical representation, where subjects were asked to manipulate and to experience the mechanical behavior of the AFM probe when it touches a sample surface. Providing haptics was hypothesized to result in a better understanding compared to providing only graphic representation. A second hypothesis was that providing the analogical representation would enhance the understanding compared to providing the realistic one, in particular for novices.

\subsection{Method}

\section{Participants}

Forty-five voluntary engineering students (41 male and 4 female), aged from 20 to 30, took part in this study ( $m e a n=24.2, s d=2.3$ ). When asked, none indicated that he/she has prior knowledge of nanoscale physics and half said to know the physics of beams. 


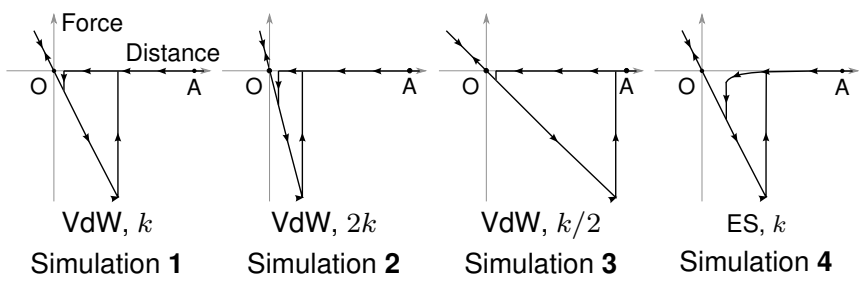

Figure 7: Force-distance curves of the four simulations.

\section{Design}

The experiment was divided in two phases. We began with a performance phase during experiential learning where subjects were asked to test successively several simulations with the interface, then to produce responses about their understanding of the AR phenomenon, and received feedback on the correctness of their responses. We created four experimental conditions combining two haptic conditions (haptics; no haptics) with two graphic conditions (cantilever; analogy), resulting in a $2 \times 2$ factorial design. Participants were randomly assigned to one of the four experimental groups in the experimental design. The second phase was devoted to the subjects' assessment of the four conditions, including the one they experienced during the performance phase.

\section{Procedure}

Subjects were seated next to the haptic device, in front of the visual display (Fig. 6). Before starting the experiment, they were trained to manipulate the haptic device. The graphic representation was then described, either as a cantilever beam with a tip or as its analogy consisting of a rigid beam, a spring, and a no-mass magnet. In the performance phase, subjects tested the AR simulation with different parameters such as the type of attraction forces and the cantilever stiffness. Simulations 1 to 3 used van der Waals forces and the following cantilever stiffness values, respectively, $k_{1}=0.2 \mathrm{~N} / \mathrm{m}$, $k_{2}=2 k_{1}$, and $k_{3}=k_{1} / 2$. Simulation 4 used electrostatic forces and the same stiffness as in Sim. $1, k_{4}=k_{1}$. The corresponding force-distance curves are illustrated in Fig. 7.

Subjects were given no time limit. They were told that they could test the current sample as long as they needed to have a clear perception of how the AR simulation behaved. Once the subjects were satisfied with the study of the simulation, their understanding of the phenomenon was evaluated. For Sim. 1, this evaluation was based on several collected data (verbal report, curve drawing and curve identification) in order to obtain a detailed depiction of what the subjects had acquired during the initial exposure to the AR simulation. Subjects were gradually asked to:

- Give verbal explanations of what they understood about the AR phenomenon;

- Draw on a paper sheet the profile of the force-distance curve they experienced; and

- Select the correct force-distance curve between eight curves of possible behavior (see Fig. 8). 

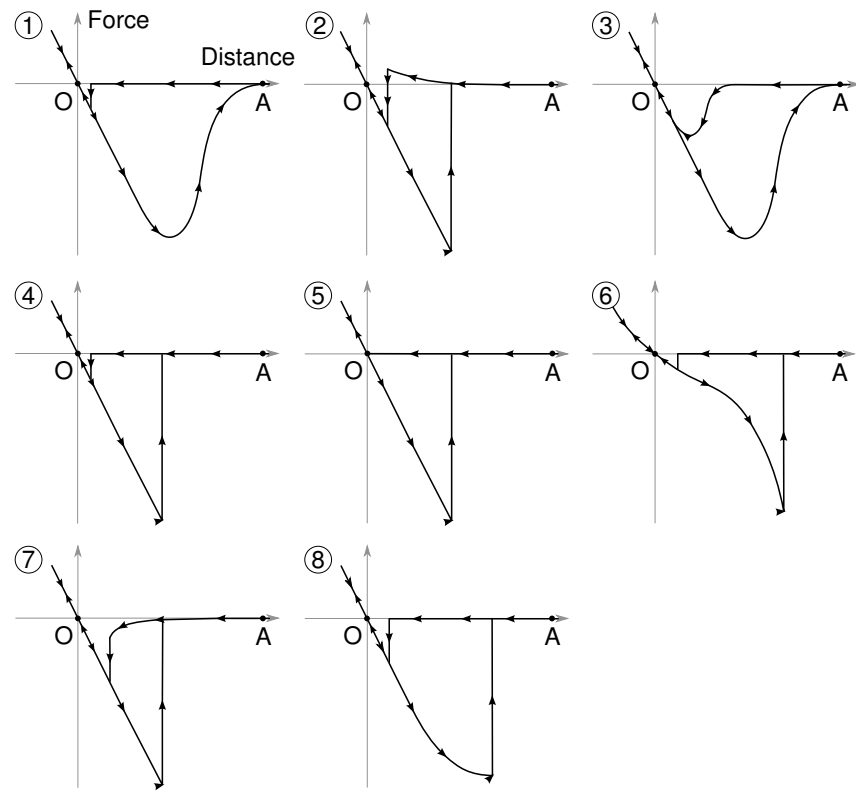

Figure 8: Force-distance curves proposed for Sim. 1.

Those three tasks also relate to different types of measures of learning: the first requires construction of a verbal representation of the phenomena; the second requires translation from sensory perceptual processes to drawings; and the third requires problem solving.

The remaining simulations were subject to a simpler evaluation. Subjects were asked to describe what differences they perceived between the current AR simulation and Sim. 1. They were then asked to identify the correct force-distance curve in a list of nine curves. To make the comparison easier, subjects were told that they could test Sim. 1 again as many times as they wanted. After each choice, the correct response was provided and, especially after the first identification, an explanation of the forces involved in the simulation was given. As engineering students, subjects had a vast experience in reading graphs.

In the second phase, subjects were asked to test the four experimental conditions successively in a random order and filled in a subjective questionnaire where they had to grade the four experimental conditions using a seven-point Likert scale, from 1 (very bad) to 7 (very good), in terms of comprehension of the AR phenomenon, perception of forces and global appreciation of the feedback. Finally, subjects were asked to rank the conditions by order of preference for an account of the AR phenomenon.

The total duration of the experiment was 55 minutes on average $\left(t_{\min }=35, t_{\max }=95\right.$, $s d=12$ ). 


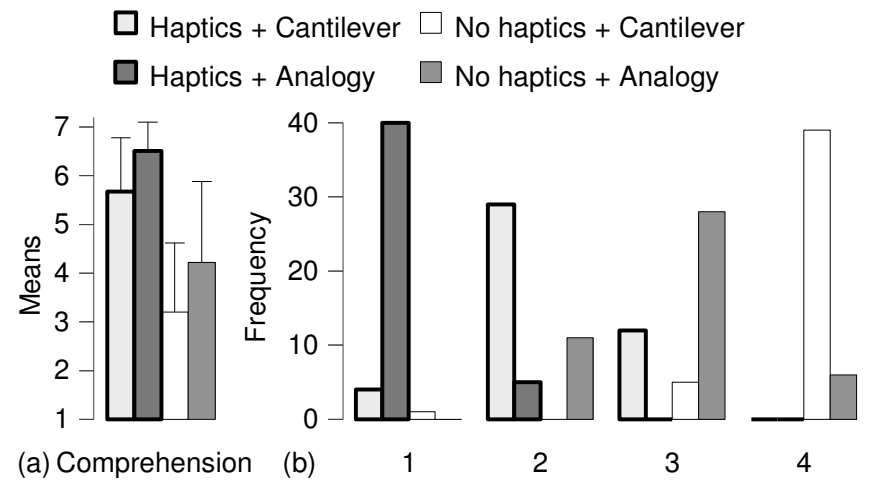

Figure 9: (a) Means of the subjective evaluation of comprehension (graded from 1 to 7). (b) Order of preference (frequency among the 45 subjects) for the four conditions regarding the case of explaining the AR phenomenon.

\section{Results}

\subsection{Preference}

The subjective evaluation of the four conditions has been addressed in (Millet et al., 2008). Results showed significant effects of both the evaluated condition and the initial group condition. Fig. 9a shows that subjects gave the best ratings for comprehension to Haptics+Analogy $(m=6.5)$ followed by Haptics+Cantilever $(m=5.7)$; the worst ratings were observed in the No-haptics+Analogy condition $(m=4.2)$ followed by No-haptics+Cantilever $(m=3.2)$.

Consistently with these results, the subjective ranking of the conditions for the potential use in explaining the AR phenomenon (see Fig. 9b) shows the best results for the Haptics conditions compared to Analogy. Nevertheless, Haptics+Analogy was the most preferred for explaining the AR phenomenon and Nohaptics+Cantilever was the least preferred. The choice between the second and the third rank was more divided between the attraction of the pedagogical graphic criterion and the haptic criterion, but finally fell in favor of the haptic criterion.

\subsection{Curve identification data}

\section{Global results}

The results for the four identifications are given in Fig. 10. Regarding the three simulations with van der Walls forces, data show that correct identification was very low for Sim. 1 (36\% correct) and increased for Sim. 2 and 3 (respectively 82\% and $91 \%$ ). Thus, only one third of the subjects identified the correct curve at this initial step whereas nearly all of them succeeded to identify the curve of Sim. 3 (Cramér's $\left.V^{2}=0.28^{2} ; \chi^{2}=37.9, D o F=2, p<0.0001\right)$. This can be seen as a learning effect

\footnotetext{
${ }^{2}$ To analyses the results, we used Cramér's criterion which measures the magnitude of the association between two nominal variables. It is conventionally considered to be weak when $V^{2}<0.04$ and strong when $V^{2}>0.16$.
} 

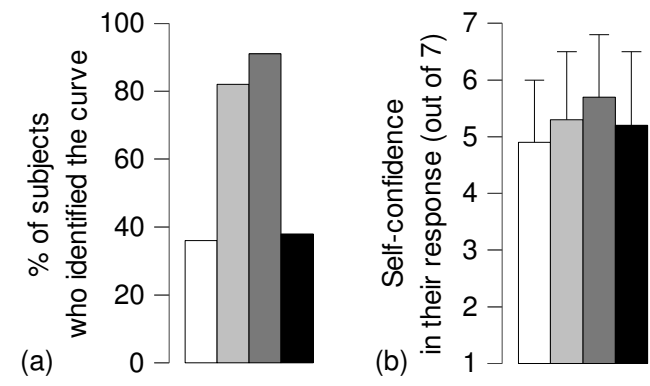

$\square$ Simulation 1
Simulation 2
Simulation 3
Simulation 4

Figure 10: Results for the curve identification: (a) frequency of correct identifications (in \%) and (b) mean self-confidence in responses given (out of 7); error bars indicate standard error of the mean for each simulation.
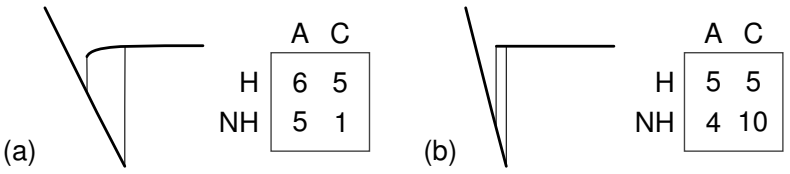

Figure 11: Main curves chosen for Sim. 4 contingent on group conditions: (a) the correct curve, (b) an incorrect one without deflection before snap-in and twice stiffer.

from Sim. 1 to $2\left(\chi^{2}=20.2, D o F=1, p<0.0001\right)$ whereas Sim. 2 and 3 exhibited a smaller (and non significant) increment in response correctness $\left(\chi^{2}=1.5, D o F=1\right.$, n.s.). Sim. 1 and 4 were specifically difficult due to respectively the absence of previous reference and a limitation of the haptic device (cf. Discussion). Note that such pattern is to be expected since Sim. 1 was the initial approach for subjects in terms of understanding the AR phenomenon. Afterwards, subjects were given a systematic feedback after each answer.

Simulation 4 , with electrostatic forces during approach, resulted in a significant decrease of the correctness, at a level similar to that observed for Sim. 1 (38\% correct). This surprising decrease in correct identification merits some comments. Subjects were torn between two curves illustrated in Fig. 11: the correct curve (17 subjects) and an incorrect one (24 subjects) that has a an early snap-in like in the correct curve, but without deflection before snap-in and with a double stiffness like in curve 2 on Fig. 7. According to the subjects' comments, they did not notice the small deflection before snap-in. Some focused their attention on the approach phase of the behavior and chose the other curve that had an earlier snap-in, without paying attention to stiffness. The others faced a conflict between a curve with a double stiffness and an earlier pull-off and one with an attraction they did not feel.

In addition, as shown by Fig. 10b, subjects were on average confident in their response on a seven-point Likert scale from 1 (highly unconfident) to 7 (highly confident), whatever the level of correctness (average values between 5 and 6 ; $s d$ about 1). 
Table 2: Numbers of correct vs. incorrect identifications contingent on visual and haptic aid provided to subjects

\begin{tabular}{lllccc}
\hline Sim. & Curve & Analogy & Cantilever & Haptics & No haptics \\
\hline \multirow{2}{*}{1} & Correct & $\mathbf{1 1}$ & $\mathbf{5}$ & 7 & 9 \\
& Incorrect & 11 & 18 & 15 & 14 \\
\multirow{2}{*}{$2 \& 3$} & Correct & $38(18+20)$ & $40(19+21)$ & $\mathbf{4 2}(21+21)$ & $\mathbf{3 6}(16+20)$ \\
& Incorrect & $6(4+2)$ & $6(4+2)$ & $2(1+1)$ & $10(7+3)$ \\
4 & Correct & 11 & 6 & 11 & 6 \\
& Incorrect & 11 & 17 & 11 & 17 \\
\hline
\end{tabular}

The bold numbers indicate significant effects.

\section{Effects of visual and haptic aids}

The results on curve identification contingent on visual and haptic aids are reported in Table 2. For the first identification, data exhibited an intermediate correlation between visual aid and correctness (Cramér's $V^{2}=0.09$ ) and a weak correlation between haptics and correctness (Cramér's $V^{2}=0.006$ ). Relative deviations (RD) measure the association between two modalities; by convention, we report only RDS with absolute values greater than 0.25 . Since half of the subjects succeeded with the Analogy condition whereas less than one quarter (22\%) succeeded with the Cantilever condition, RDS accordingly show that successful identifications were correlated with the Analogy representation $(\mathrm{RD}=0.41)$ and anticorrelated with the Cantilever representation $(\mathrm{RD}=-0.39)$. Further tests showed a marginally significant effect for visual aid (Fisher's test, $p=0.065$ ) and no significant effect for haptic aid (Fisher's test, $p=0.76$ ).

We aggregated curve identification data for Sim. 2 and 3, which focused on the influence of the cantilever stiffness. In contrast to Sim. 1, we observed a mild correlation between haptics and correctness (Cramér's $V^{2}=0.06$ ) and a weak correlation between visual aid and correctness (Cramér's $V^{2}=0$ ). Relative deviations essentially show that incorrect identifications were anticorrelated with Haptics $(\mathrm{RD}=-0.65)$ and correlated with No-haptics $(\mathrm{RD}=0.60)$. Further tests showed a significant effect for haptic aid (Fisher's test, $p=0.029$ ) and no significant effect for visual aid (Fisher's test, $p=1$ ).

For Sim. 4 with electrostatic forces, data exhibited an intermediate correlation between haptics and correctness (Cramér's $V^{2}=0.06$ ) as well as between visual aid and correctness (Cramér's $V^{2}=0.06$ ). Relative deviations mostly emphasized that correct identifications were correlated with Haptics $(\mathrm{RD}=0.32)$ and anticorrelated with the Nohaptics conditions $(\mathrm{RD}=-0.31)$. The same pattern was found for the positive association between the Analogy representation and correctness $(\mathrm{RD}=0.32)$ and the negative association between the Cantilever representation and correctness ( $\mathrm{RD}=-0.31)$. However, further tests showed no significant effect for both haptic (Fisher's test, $p=0.23$ ) and visual aids (Fisher's test, $p=0.23$ ).

\section{Synthesis}

This first set of results suggests that during the initial exposure to the AR phenomenon, the magnet-spring analogy can help to make the link between the phenomenon and its force-distance curve, while haptic information provided no evidence of contribution 

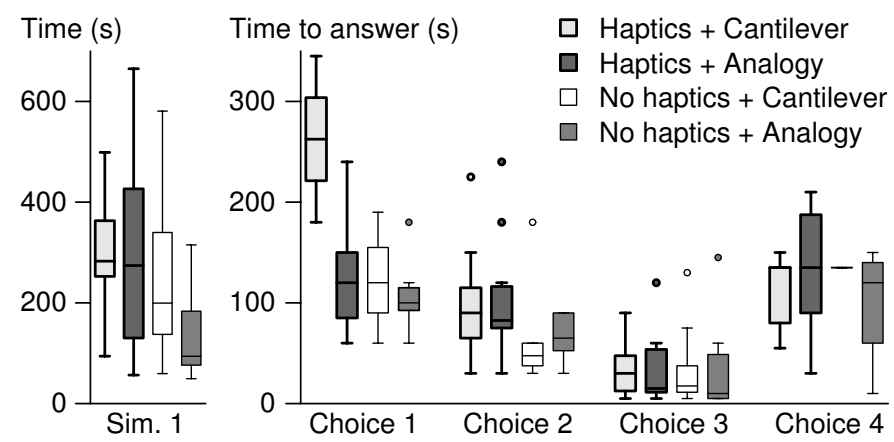

Figure 12: Time spent (a) testing the first simulation and (b) identifying the curve in each simulation, restricted to the subjects who chose the correct curve.

to subjects' understanding. During Sim. 2 and 3 exploring the effect of the stiffness on the curve, providing haptics to a group of subjects made this group significantly more skillful. For Sim. 4 with different attraction forces, trends suggest that both haptics and the analogy could have a positive influence on this identification in that subjects underperformed in the condition associating no haptics with the cantilever representation. However, the latter results need to be further investigated.

\subsection{Time data}

\section{Global results}

Subjects took longer to choose the curve for the first simulation $(m=169$ s, i.e., about 3 minutes) than for the others (respectively 90, 35 and $90 \mathrm{~s}$ ). The durations were significantly different between identifications 1 and 2 (paired $t$-test $=5.82, D o F=44$, $p<.0001$ ) as well as between identifications 2 and 3 (paired $t$-test $=8.62, D o F=44$, $p<.0001$ ) since subjects responded increasingly quickly. The introduction of electrostatic forces in Sim. 4 was clearly accompanied by a significant increase in time taken by subjects to answer (paired $t$-test $=-4.42, D o F=44, p<.0001$ ).

\section{Effects of visual and haptic aids}

The time spent by subjects on testing the first simulation and then on each curve identification is given in Fig. 12. Regarding the duration of testing the first simulation, we computed an analysis of variance (ANOVA) with visual and haptic aids as mixed between-subject factors. A significant main effect of Haptics was found $(F(1,41)=5.50$, $p<0.024)$, whereas there was no significant effect of Graphic representation $(F(1,41)=$ 1.44 , n.s.), nor was two-way interaction between the two factors found $(F(1,41)=1.34$, n.s.). As indicated in Table 3, subjects spent indeed about $50 \%$ more time in testing and interacting with the simulation when provided with haptic feedback. The same pattern was found for the drawing time, with a significant effect of Haptics $(F(1,41)=11.7$, $p<.0014)$ but no significant effect of Graphic representation $(F(1,41)=0.05$, n.s. $)$ or for the two-way interaction $(F(1,41)=2.77$, n.s. $)$.

Subsequent analyses of time data are restricted to the subjects who chose the correct curve (see Table 3). For the first identification, the 16 subjects who correctly answered 
Table 3: Mean (sd) time in seconds spent by subjects, for testing Sim. 1, for drawing the curve, and for identifying the curve (restricted to the subjects who correctly answered), contingent on visual and haptic aids

\begin{tabular}{lcccc}
\hline & Analogy & Cantilever & Haptics & No haptics \\
\hline Testing Sim. 1 & $221(182)$ & $275(139)$ & $\mathbf{3 0 3}(167)$ & $\mathbf{1 9 7}(142)$ \\
Drawing Sim. 1 & $243(151)$ & $250(92)$ & $\mathbf{3 0 3}(133)$ & $\mathbf{1 9 2}(85)$ \\
Identif. Sim. 1 & $\mathbf{1 1 9}(54)$ & $\mathbf{1 7 9}(106)$ & $\mathbf{1 6 9}(98)$ & $\mathbf{1 1 3}(46)$ \\
Identif. Sim. 2 & $86(53)$ & $83(53)$ & $\mathbf{1 0 0}(57)$ & $\mathbf{6 3}(38)$ \\
Identif. Sim. 3 & $33(40)$ & $35(34)$ & $35(32)$ & $33(41)$ \\
Identif. Sim. 4 & $115(66)$ & $115(38)$ & $122(58)$ & $103(55)$ \\
\hline
\end{tabular}

The bold numbers indicate significant effects.

took 50\% more time with haptics than without, whereas they took $34 \%$ less time with the Analogy than with the Cantilever representation. The two-way ANOVA showed a significant main effect of Haptics $(F(1,12)=5.27, p<0.04)$ and a nearly significant trend of Graphic representation $(F(1,12)=4.32, p<0.06)$ but no effect for the two-way interaction $(F(1,12)=2.73$, n.s. $)$.

For Sim. 2, the subjects who correctly answered (37 out of 45 ) took 59\% more time with haptics than without whereas they took almost the same time whatever the graphic representation. We observed a significant effect of Haptics $(F(1,33)=4.81$, $p<0.036)$ and no significant effect of Graphic representation $(F(1,33)=0.07$, n.s. $)$ or for the two-way interaction $(F(1,33)=0.01$, n.s. $)$.

Regarding Sim. 3, characterized with the highest level of correct identification (41 out of 45), the subjects took almost the same time whatever the condition. The high variability within groups combined with the small differences in mean led to no significant effect of Haptics $(F(1,37)=0.03$, n.s. $)$, of Graphic representation $(F(1,37)=0.03$, n.s.), or for the two-way interaction $(F(1,37)=0.00$, n.s.).

For Sim. 4 with electrostatic forces, the 17 subjects who correctly answered took more time with haptics than without whereas they took almost the same time whatever the graphic representation. However, statistical tests showed no significant effect of Haptics $(F(1,37)=0.02$, n.s. $)$, of Graphic representation $(F(1,37)=0.06$, n.s. $)$, as well as for the two-way interaction $(F(1,13)=0.63$, n.s. $)$.

\section{Synthesis}

This second set of results shows that visual and haptic aids affected the early phases of responses during the study. Haptics induced a significant increase in the time spent to test and to explore the simulation as well as in the drawing time and in the time to select the correct curve at Sim. 1 and 2. Several complementary explanations may be proposed. First, the provision of an interactive haptic device can generate more motivation and engagement to achieve a deeper understanding during the task. Second, haptics can orient the exploration and focus subject's attention towards task-relevant force information that is considered less in the conditions without haptics (possibly due to a modal-bias effect as shown by Lederman and Klatzky (2004) in an object classification task). Third, adding haptics may increase the amount of processed information to achieve the task. Fourth, the integration of both visual and haptic information into the mental model developed by the subjects can add a supplementary cost. 


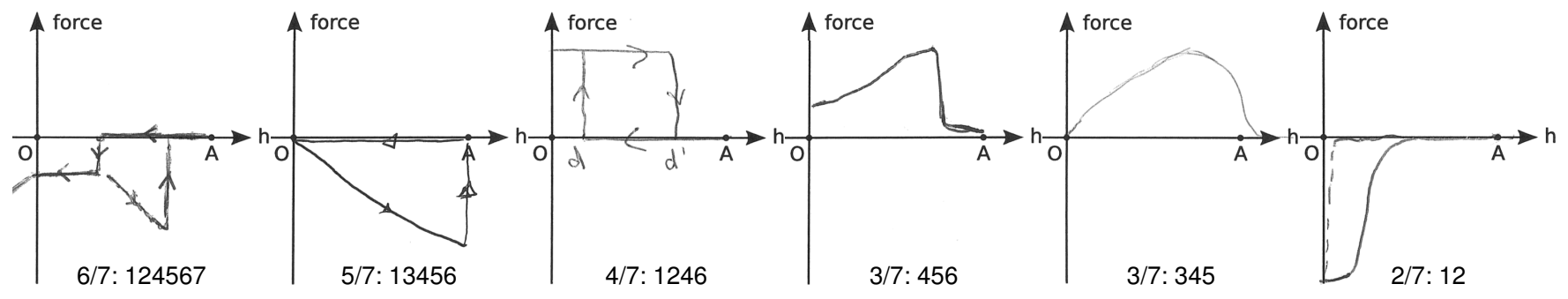

Figure 13: Samples of subjects' drawings. The grade and criteria validated are reported for each sample.

Table 4: Criteria used for evaluating the accuracy of drawings

\begin{tabular}{cll}
\hline Criterion & Drawing property & Physical meaning \\
\hline 1 & A-O-A & Two-way curve (hysteresis) \\
2 & Vertical BC & Snap-in instability \\
3 & Go through O & Minimum energy of the system \\
4 & Straight OD & Linear cantilever elasticity \\
5 & Growing $|\mathrm{OD}|$ & Adhesion tip - substrate \\
6 & Vertical DE & Pull-off instability \\
7 & BC $<$ DE & Snap-in force $<$ pull-off force \\
\hline
\end{tabular}

Regarding multimodal integration, it has been showed that the more multimodal the signal is, the shorter the response time is in detection tasks such as temporal detection and locating of a multimodal signal (Hecht et al., 2006, 2008a,b). In the context of multimodal integration in mental model, however, this is not straightforward because links have to be established between the different sensory representations; furthermore, multimodal learning environment generate a heavy cognitive load that is detrimental to learning.

We also observed a trend that the provision of an analogical representation decreases the response time at Sim. 1 curve identification, suggesting that useful information could be exploited more efficiently with this representation than with the Cantilever representation. This potential effect would require further investigation.

\subsection{Subjects' drawings}

Prior to the curve identification step, subjects were asked to draw their own curve based on their perception of the phenomenon. This measure of initial understanding can be investigated by looking at the accuracy of the drawings produced by subjects. The drawings were analyzed and rated according to seven criteria listed in Table 4. These criteria correspond to the main properties of a force-distance curve as described in Section 3.1. Some samples of drawings are reported in Fig. 13.

The accuracy of drawings is reported in Table 5. Globally, all subjects and conditions taken together, the quality of drawings was on average 3.0 out of $7 ; s d=2.1$. On the one hand, the subjects who correctly identified the curve were actually better in the drawing test $(m=3.9, s d=.51)$ than those who failed $(m=2.5, s d=.38$; $t(43)=-2.35, p<.012)$. On the other hand, the ANova revealed no significant effect 
Table 5: Mean (sd) accuracy of drawings

\begin{tabular}{lccc}
\hline & Analogy & Cantilever & All \\
\hline Haptics & $3.9(2)$ & $3.0(2)$ & $3.5(2)$ \\
No haptics & $2.3(2)$ & $2.8(2)$ & $2.5(2)$ \\
All & $3.1(2)$ & $2.9(2)$ & $3.0(2)$ \\
\hline
\end{tabular}

of Haptics $(F(1,41)=2.21$, n.s. $)$, of Graphic representation $(F(1,41)=0.12$, n.s. $)$, or for the two-way interaction $(F(1,41)=1.19$, n.s.), partly due to the high variability observed within groups.

Taken together, these results suggest that learning is an active and incremental process affected by several factors, both internal and external, whose effect is difficult to isolate in a single step. Our data show that the better the model of AR was early constructed as testified by the drawing performance, the higher the probability to correctly identify the curve of Sim. 1. Simultaneously, neither haptics nor visual aid had a sufficient effect to significantly impact the accuracy of drawings produced by subjects, although visual aid significantly affected the correctness of the curve identification of Sim. 1.

\subsection{Mental models developed by subjects}

A consequence of the results reported in the previous sections is that several indicators are necessary to get a rich and complete picture of the processes involved in understanding and learning activities. Indeed, different conclusions would be drawn if analyses were restricted to only one single - and quite unidimensional-numerical indicator.

To further explore the content and processes related to mental models built by subjects during their tasks, we looked at potential correlations between subjects' performance, verbal productions about the phenomenon and experimental conditions. To address this issue, we conducted a Principal Component Analysis (PCA) to underline the potential geometric underlying structure of the set of partially correlated quantitative and qualitative data, including verbal data produced by subjects while they were testing the first AR simulation.

The analysis was based on the following set of data. Performance measures were: correctness in identifying the curve for Sim. 1 and 4, drawing time, and drawing correctness. The subjects' explanations written during Sim. 1 were coded according to the presence of verbal indicators of different themes used to describe the AR phenomenon. A theme was scored 1 when at least one of its verbal indicators was present in the explanation. Some of the themes were discarded afterward from the PCA due to their low frequency and/or their lack of discrimination power between subjects. The verbal indicators of the themes are summarized in Table 6. Last, experimental conditions added two qualitative variables: haptic and graphic conditions.

The PCA was computed with the Stata 10.1 software package. As can be seen in Table 7 , we retained four factors, corresponding to $66 \%$ of the variance explained, as they provide an interesting insight about the correlations between mental models, performances and conditions. All the variables are well explained by the four factors 
Table 6: Verbal indicators found in the descriptions provided by subjects and retained for the principal component analysis

\begin{tabular}{ll}
\hline Label & Verbal indicators \\
\hline Cantilever & Cantilever, tip, cone \\
Analogy & Magnet, magnetic, spring, capillarity \\
Proportionality & Proportional, linear spring \\
Adhesion & Adhesion, stuck, fixed, locked, attached \\
Dynamics & Snap off, sudden, shock \\
Active haptic & To apply, to force, to pull \\
\hline
\end{tabular}

Table 7: Principal component analysis_-factor loadings, uniquenesses, eigenvalues, and proportions for the four first factors

\begin{tabular}{lrrrrr}
\hline Variable & Factor 1 & Factor 2 & Factor 3 & Factor 4 & Uniq. \\
\hline Identification 1 & -.19 & $\mathbf{. 6 3}$ &. $\mathbf{4 4}$ & -.13 & .36 \\
Identification 4 &. $\mathbf{4 9}$ &.$- \mathbf{4 2}$ & -.17 &.$- \mathbf{5 4}$ & .25 \\
Drawing time &. $\mathbf{7 4}$ & .21 & .23 & .23 & .31 \\
Drawing score & .29 &. $\mathbf{4 4}$ &. $\mathbf{5 3}$ & .22 & .39 \\
Cantilever & .34 & -.01 & -.18 & .77 & .26 \\
Analogy & -.16 &. $\mathbf{7 3}$ & -.13 & -.02 & .42 \\
Proportionality & -.05 & .05 & .77 & -.32 & .31 \\
Adhesion & -.18 & -.70 & .09 & .17 & .43 \\
Dynamics & .08 & -.28 &. $\mathbf{6 6}$ & -.10 & .46 \\
Active haptics &. $\mathbf{7 8}$ & -.16 & -.34 & .01 & .25 \\
Graphic condition & -.03 & -.21 & -.15 &. $\mathbf{8 1}$ & .28 \\
Haptic condition &. $\mathbf{7 6}$ & -.07 & .23 & .04 & .37 \\
\hline Eigenvalue & 2.27 & 2.00 & 1.84 & 1.80 & \\
Proportion & .19 & .17 & .15 & .15 & \\
\hline
\end{tabular}

(uniqueness is less than 0.6). Axes are interpreted using the variables with the highest factor loadings (absolute value greater than 0.4).

\section{Interpretation}

Axis 1 (22\% of variance explained) contrasts, on the one hand, the subjects who tended to find the correct curve of Sim. 4 (with electrostatic forces) with, on the other hand, those who did not. The first group corresponded mostly to conditions with haptics, mentioned the kinesthetic information perceived and took more time in drawing. Interestingly, this axis does not separate the subjects who found the curve of Sim. 1 from those who did not, suggesting that all these characteristics did not affect the initial understanding, as it was observed previously. This axis can be interpreted as illustrating the specific contribution of haptics, which results in a trend to focus understanding on forces-which can help in Sim. 4 to compare the stiffness with that in Sim. 1-and increases the time taken to process information.

Axis $2(18 \%)$ contrasts the subjects who found the correct curve of Sim. 1, produced correct drawings but also tended to fail in Sim. 4. Interestingly, this group is characterized as using analogies to describe the AR phenomenon, due to either the Analogy representation or analogies spontaneously generated (mainly analogies with a magnet or a spring for the subjects with the Cantilever representation). This suggests 
that constructing a mental model to explain the AR phenomenon by analogy would favor success in the first curve identification and can be considered as a possible strategy, whatever the graphic representation provided. This strategy is also suggested by the fact that this axis does not relate to any particular experimental condition. In terms of structure mapping (Gentner, 1983), previous knowledge in the base domain (analogy) that could favor this strategy is the decreasing attraction force of a magnet or the straight force-distance curve of a spring. Furthermore, this group did not mention adhesion effects, which may be due to the obviousness of the adhesion behavior of the magnet in the magnet-spring analogy.

Axis $3(16 \%)$ contrasts, on the one hand, the subjects who tended to accurately answer in Sim. 1, when finding the correct curve and producing correct drawings, with, on the other hand, those who did not. This group is also characterized by the mention of aspects of proportionality and pull-off dynamics, which suggests that noticing these aspects favors success in Sim. 1. Like axis 2, this axis shows no correlation with experimental conditions, which suggests a complementary dimension of the construction of mental models to succeed in Sim. 1.

Axis $4(11 \%)$ contrasts the subjects who failed to find the correct curve of Sim. 4 with those who succeeded. The former group were mostly with the Cantilever representation and mentioned the terms related to this representation. Oppositely, the other group was characterized as using the Analogy representation and having limited failure in Sim. 4.

\section{Synthesis}

These four axes provide a consistent view of cognitive processes and factors related to novices testing simulations to understand several dimensions of the AR phenomenon. They complete the performance data previously analyzed by suggesting a global frame for interpreting our pattern of results. Indeed, axes 1 and 4 clearly represent the effect of respectively the haptic and the graphic conditions on performance and features of the mental models. The presence of haptics increased the time taken for drawing the curve, yet without significantly improving its accuracy, and helped the subjects to compare cantilever stiffnesses or pull-off positions in Sim. 4. Likewise, the threshold marks in the Analogy representation helped to compare pull-off positions in Sim. 4.

In contrast, axes 2 and 3 are not associated with the haptic and graphic experimental factors. Both axes can be interpreted as representing two complementary ways followed when constructing a mental model to explain AR phenomenon from the simulation, and leading to make correct drawings and right choices. Axis 2 shows a first way by reasoning by analogy, which may be induced either by the Analogy representation or, for some subjects, by a spontaneously generated analogy, presenting a similar effect on understanding of AR phenomenon. Axis 3 shows another way by noticing both aspects of proportionality of the force/distance ratio during contact and of dynamics of the AR behavior-two aspects that represent two important features of the curve.

\section{General discussion}

In terms of preference, haptics was globally the first criterion for the subjects, who globally were more influenced by the presence of haptics than by the analogical repre- 
sentation. From the four conditions tested in our study, the condition with haptics and the analogy was the most preferred however.

We found that haptic feedback and the analogical representation seemed to positively influence the learning process at different phases. Haptics brought an improvement in the subsequent phases whereas the analogy could have a fostering effect at the time of the first exposure to the simulation.

Haptics can generate more engaging experiences, make the students' explanations focus on forces and facilitate the extraction of task-relevant force information

Haptics proved to benefit to subjects. From a mere performance perspective, subjects in the haptic conditions exhibited a better understanding than the groups without haptics only after the first simulation. This superiority disappeared when introducing electrostatic forces as a new parameter in Sim. 4. This lack of effect of haptics at the first simulation may be however due to the characteristics of the experimental material, and especially of an interaction between characteristics of haptic and visual feedback used in our simulation (cf. discussion below). We also found that the groups with haptics took more time for testing, drawing, and answering in the early phases of the exposure to the simulation. We have advanced several possible explanations to this phenomena. The reason could be that the simulation was more engaging with haptics.

Haptic feedback can also orient the exploration and focus subjects attention towards task-relevant forces information that are less considered in the conditions without haptics (possibly due to a modal-bias effect as shown by Lederman and Klatzky (2004) in a object classification task). Consistently with this explanation, the PCA showed that haptic feedback tended to focus the students' explanations on forces, like in (Persson et al., 2007) where force feedback focused understanding on the forces involved in the docking process. By focusing attention on forces, haptic feedback proved thus useful for perceiving the effect of the cantilever stiffness. This type of result is rather similar to that in (Bussell, 2004) where force feedback helped fifth-grade students to understand the effect of increased gravity in a computer-based paddleball simulation that taught concepts of gravity, mass, force and motion. In the context of nanoscience education where comprehending nanoscale forces is challenging, haptic technology can help learners to grasp the notions of force at a distance and spatial force threshold.

It is worth noting that in an educational context where students would be given more information about the interpretation of the haptic information, the longer time observed in our study may be largely reduced. Finally, adding haptics may also increase the amount of information subjects must process to achieve the task. These results should be replicated however, and further work is needed to improve our understanding of the role of haptics in VR environment for learning and understanding concepts of physics at the nanoscale.

\section{Analogy could improve understanding and learning at the first step}

The magnet-spring representation, preferred by subjects, had a nearly significant effect at the discovery-stage of the AR behavior, with a better identification of the curve and in a shorter time. In addition, one third of the subjects of the groups with the cantilever representation spontaneously elaborated, orally or in writing, the magnetspring analogy. These subjects obtained a level of correct identification similar to that 
of the subjects with the analogy representation, i.e., two times greater than without reasoning by analogy. This is in line with previous studies on spontaneous analogies (Kaufman et al., 1996; Sandifer, 2004). The PCA also showed that subjects who saw or thought of the analogy produced better drawings. Although little literature has been found about the value of drawings in physical experiments, drawings were used in some works to study subjects' understanding (Jones et al., 2003; Marchi et al., 2005). Overall, the data suggests that the magnet-spring analogy could be useful for explaining to people without any prior knowledge the AR phenomenon and the link with its forcedistance curve.

Graphic representations cue students to focus on particular characteristics of physical phenomena (Podolefsky and Finkelstein, 2006). Here, the magnet-spring representation aims at explaining the hysteretic behavior, by focusing on the combination of linear elasticity and decreasing attraction force. Nevertheless, the physical origin is different, magnetic forces in this analogy do not reproduce the plurality in type and in range of nanoscale forces. Also, the analogy was presented to subjects as a dynamic simulation. Using only text and static pictures of the same analogy would probably weaken the benefits for student learning (Trey and Khan, 2008). Provided that the magnet-spring analogy is presented as a dynamic simulation or device, this analogy appears to be a good candidate for AFM teaching in an introductory course,

More generally, the magnet-spring analogy could help understanding the link between hysteretic behavior and any linear deformable system interacting with adhesion forces or decreasing nonlinear attraction forces. It could also be used as a mechanical example of any hysteretic behavior, whether it is electronic, magnetic, elastic, etc.

\section{Effects of combining visual and haptic feedback}

In the early phase of understanding, our study has shown that the combined use of haptics and the magnet-spring analogy could be a way to reduce the extra processing time generated by the use of haptics with visual representation. As a possible explanation for the longer time observed for subjects with haptic feedback, we have indeed suggested that the integration of both visual and haptic information could add supplementary loads to the building of the mental model developed by the subjects.

When identifying the first curve, we observed a specific pattern effect between force feedback and the cantilever representation. Among the wrong answers, reported in Fig. 14, it is noticeable that curve 6 was chosen the most often in the Haptic+Cantilever condition. This curve had a nonlinear slope (increasing with the distance) during retract. According to these subjects' comments about their incorrect answer, such as "I had a nonlinear feeling" or "I thought that if the force goes up then it's not linear", they translated the raising force they felt in an increasing slope. This incorrect interpretation of force feedback did not occur in the other conditions. This could be due to the association of focusing on forces and viewing the cantilever bending (in a nonlinear shape), which can be seen as a visual pseudo-haptic effect (Lécuyer, 2009).

A limitation appeared with the attraction before snap-in presented in Sim. 4, which proved difficult to feel on our platform. The deflection was barely above the mechanical noise of the device and subjects were not asked to apply finer discrimination at that point. That is a limitation that should be reduced for an educational application. For that, several ways can be considered for improving the perception of these small 


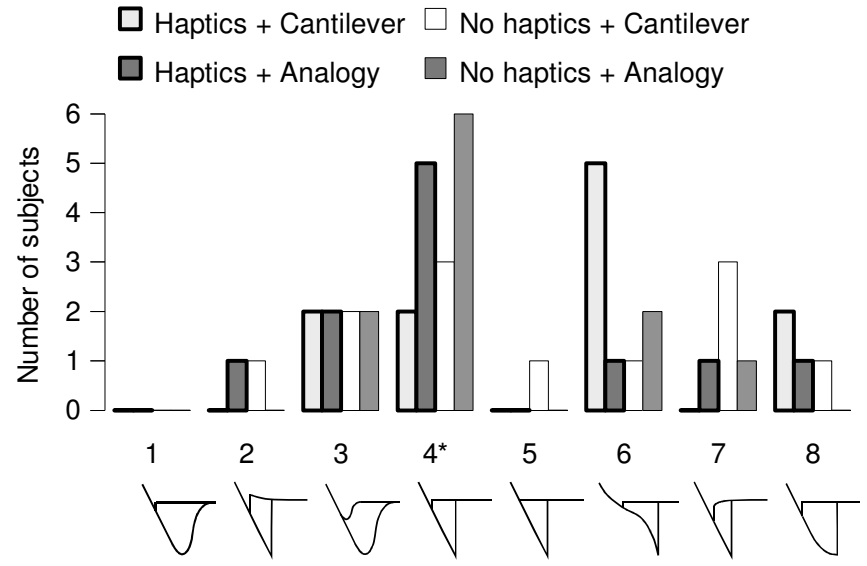

Figure 14: Number of identifications for simulation 1 as function of the four group conditions. The * indicates the correct answer.

but fast-rising forces and deformations while rendering the whole hysteresis cycle. Visually, moving the substrate towards the probe rather than the opposite should facilitate the detection of the small deformations, as the frame of the deformations would be fixed. Similarly, a graphical cue such as the shape of an undeformed cantilever would make the comparison with the deformed shape easier. Haptically, using a higherfidelity haptic device could refine the haptic rendering (Mohand Ousaid et al., 2011). Perhaps a better solution may be sound feedback as proposed by Marchi et al. (2005).

Although prior knowledge of nanoscale physics was asked to the subjects, the study lacks formal pre-assessments of the subjects' knowledge of relevant concepts and skills such as nanoscale physics, mechanics at the macro-scale, beam theory, or graph reading ability. A consequence is that some subjects might have some facilitating knowledge or misconception prior to their exposition to the phenomena and the use of the simulator. It is however difficult to evaluate the potential impact since this could either facilitate or hamper the knowledge construction process.

Lastly, although subjects did not perform better with haptics in the tests of initial understanding, it would be interesting for future work to study the effect of haptics on the learning of new knowledge in the long term by means of longitudinal studies over one month or one year: would subjects who discovered the AR phenomenon with haptic feedback internalize and memorize it better?

\section{Conclusion}

This paper described an experimental platform set up to evaluate the use of force feedback and a graphic magnet-spring analogy for understanding the approach-retract cycle of an AFM probe, for people without prior knowledge. The results showed that both parameters studied were appreciated by subjects and had an influence on their perception and their understanding of the approach-retract phenomenon. 
The addition of haptic feedback increased the attention on forces involved in the AR phenomenon and improved the perception of the influence of physical parameters, by providing more information. The magnet-spring analogy helped subjects in early phases of understanding to link the AR phenomenon to its force-distance curve and seems therefore to be a good candidate for teaching AFM techniques in an introductory course. Taken together, our results suggest that future teaching aids for nanoscale phenomena could combine both haptic feedback and graphic analogies.

\section{Acknowledgement}

This work was supported by the French National Research Agency (ANR), through the "Perception haptique des échelles micro et nanoscopiques" (PACMAN) project. The authors would like to thank Vincent Hayward and the reviewers for their insightful comments and criticisms, and all participants who took part in this study.

\section{References}

Baddeley, A., Jan. 1992. Working memory. Science 255 (5044), 556-559.

Bannert, M., 2002. Managing cognitive load-recent trends in cognitive load theory. Learning and Instruction 12 (1), 139-146.

Bolopion, A., Xie, H., Haliyo, S., Régnier, S., 2012. Haptic teleoperation for 3d microassembly of spherical objects. IEEE/ASME Transactions on Mechatronics 17 (1), $116-127$.

Brooks, Jr., F. P., Ouh-Young, M., Batter, J. J., Kilpatrick, P. J., Aug. 1990. Project grope-haptic displays for scientific visualization. SIGGRAPH Computer Graphics 24 (4), 177-185.

Bruner, J. S., 1966. Toward a Theory of Instruction. Cambridge, MA: Harvard University Press.

Burkhardt, J.-M., Lourdeaux, D., Mellet d'Huart, D., 2003. La conception des environnements virtuels pour l'apprentissage. In: Fuchs, P., Moreau, G. C. (Eds.), Le traité de la réalité virtuelle. Vol. 2. Presses de l'école des Mines, Paris, Ch. 7, pp. 207-296.

Bussell, L., Jun. 2004. Force feedback and student reasoning. In: Proceedings of the 3rd Conference on Interaction Design and Children (IDC'04). ACM, Maryland, NY, USA, pp. 117-118.

Bétrancourt, M., Tversky, B., 2000. Effect of computer animation on user's performance: a review. Le travail humain 63 (4), 311-329.

Carpick, R. W., Ogletree, D. F., Salmeron, M., Mar. 1999. A general equation for fitting contact area and friction vs load measurements. Journal of Colloid and Interface Science 211 (2), 395-400. 
Chan, M. S., Black, J. B., Jul. 2006a. Direct-manipulation animation: incorporating the haptic channel in the learning process to support middle school students in science learning and mental model acquisition. In: Proceedings of the 7th International Conference on Learning sciences, ICLS '06. International Society of the Learning Sciences, Bloomington, Indiana, pp. 64-70.

Chan, M. S., Black, J. B., Apr. 2006b. Learning newtonian mechanics with an animation game: The role of presentation format on mental model acquisition, paper presented at the Annual Meeting of the American Educational Research Association (AERA).

Clement, J., 1988. Observed methods for generating analogies in scientific problem solving. Cognitive Science 12 (4), 563-586.

Duit, R., Roth, W.-M., Komorek, M., Wilbers, J., 2001. Fostering conceptual change by analogies-between scylla and charybdis. Learning and Instruction 11 (4-5), 283303.

Ferreira, A., Mavroidis, C., Sep. 2006. Virtual reality and haptics for nanorobotics. IEEE Robotics and Automation Magazine 13 (3), 78-92.

Finkelstein, N. D., Adams, W. K., Keller, C. J., Kohl, P. B., Perkins, K. K., Podolefsky, N. S., Reid, S., LeMaster, R., Oct. 2005. When learning about the real world is better done virtually: A study of substituting computer simulations for laboratory equipment. Phys. Rev. ST Phys. Educ. Res. 1 (1), 010103.

Gaißert, N., Wallraven, C., Bülthoff, H. H., 2010. Visual and haptic perceptual spaces show high similarity in humans. Journal of Vision 10 (11), 1-20.

Gauthier, M., Régnier, S., Rougeot, P., Chaillet, N., 2005. Analysis of forces for micromanipulations in dry and liquid media. Journal of Micromechatronics 3 (3), 389413.

Gentner, D., 1983. Structure-mapping: A theoretical framework for analogy. Cognitive Science 7 (2), 155-170.

Gentner, D., 1989. The mechanisms of analogical learning. In: Vosniadou, S., Ortony, A. (Eds.), Similarity and Analogical Reasoning. Cambridge University Press, Ch. 7, pp. 199-241.

Greczyło, T., Debowska, E., 2006. The macroscopic model of an atomic force microscope in the students' laboratory. European Journal of Physics 27 (3), 501.

Grow, D. I., Verner, L. N., Okamura, A. M., Mar. 2007. Educational haptics. In: Proceedings of the AAAI Spring Symposium: Semantic Scientific Knowledge Integration. Stanford, CA, USA, pp. 53-58.

Haliyo, D. S., Régnier, S., Guinot, J.-C., 2003. [mü]mad, the adhesion based dynamic micro-manipulator. European Journal of Mechanics - A/Solids 22 (6), 903-916. 
Hecht, D., Reiner, M., Halevy, G., 2006. Multimodal virtual environments: Response times, attention, and presence. Presence 15 (5), 515-523.

Hecht, D., Reiner, M., Karni, A., Mar. 2008a. Enhancement of response times to bi- and tri-modal sensory stimuli during active movements. Experimental Brain Research $185(4), 655-665$.

Hecht, D., Reiner, M., Karni, A., Aug. 2008b. Multisensory enhancement: gains in choice and in simple response times. Experimental Brain Research 189 (2), 133143.

Hegarty, M., Jun. 2004. Dynamic visualizations and learning: getting to the difficult questions. Learning and Instruction 14 (3), 343-351.

Hingant, B., Albe, V., 2010. Nanosciences and nanotechnologies learning and teaching in secondary education: a review of literature. Studies in Science Education 46 (2), 121-152.

Israelachvili, J. N., 1992. Intermolecular \& Surface Forces, 2nd Edition. Harcourt Brace \& Company.

Johnson-Laird, P. N., 1983. Mental models: towards a cognitive science of language, inference, and consciousness. Harvard University Press, Cambridge, MA, USA.

Jones, M. G., Andre, T., Superfine, R., Taylor, R., 2003. Learning at the nanoscale: The impact of students' use of remote microscopy on concepts of viruses, scale, and microscopy. Journal of Research in Science Teaching 40 (3), 303-322.

Jones, M. G., Minogue, J., Tretter, T. R., Negishi, A., Taylor, R., Jan. 2006. Haptic augmentation of science instruction: Does touch matter? Science Education 90, 111-123.

Kaufman, D. R., Patel, V. L., Magder, S. A., 1996. The explanatory role of spontaneously generated analogies in reasoning about physiological concepts. International Journal of Science Education 18 (3), 369-386.

Kintsch, W., Apr. 1988. The role of knowledge in discourse comprehension: A construction-integration model. Psychological Review 95 (2), 163-182.

Lederman, S., Klatzky, R., 2009. Haptic perception: A tutorial. Attention, Perception, \& Psychophysics 71, 1439-1459, 10.3758/APP.71.7.1439.

Lederman, S. J., Klatzky, R. L., Jul. 1987. Hand movements: a window into haptic object recognition. Cognitive Psychology 19 (3), 342-368.

Lederman, S. J., Klatzky, R. L., May 2004. Haptic identification of common objects: effects of constraining the manual exploration process. Perception \& Psychophysics 66 (4), 618-628.

Lederman, S. J., Summers, C., Klatzky, R. L., 1996. Cognitive salience of haptic object properties: role of modality-encoding bias. Perception 25 (8), 983-998. 
Li, G., Xi, N., Chen, H., Pomeroy, C., Prokos, M., Sep. 2005. Videolized atomic force microscopy for interactive nanomanipulation and nanoassembly. IEEE Transactions on Nanotechnology 4 (5), 605-615.

Lécuyer, A., Jan. 2009. Simulating haptic feedback using vision: A survey of research and applications of pseudo-haptic feedback. Presence: Teleoper. Virtual Environ. 18 (1), 39-53.

Marchi, F., Marliere, S., Urma, D., Florens, J.-L., Chevrier, J., Cadoz, C., Luciani, A., Jun. 2005. Interactive learning of nanophysics phenomena. In: Proceedings of the m-ICTE2005. Cáceres, Spain, pp. 510-515.

Marlière, S., Urma, D., Florens, J.-L., Marchi, F., Jun. 2004. Multi-sensorial interaction with a nano-scale phenomenon: the force curve. In: Proceedings of the EuroHaptics 2004. Munich, Germany, pp. 246-252.

Mayer, R. E., 2001. Multimedia Learning. Cambridge University Press.

Millet, G., Lécuyer, A., Burkhardt, J., Haliyo, S., Régnier, S., Jun. 2008. Improving perception and understanding of nanoscale phenomena using haptics and visual analogy. In: Haptics: Perception, Devices and Scenarios. Proceedings of the 6th International Conference, EuroHaptics 2008. Vol. 5024 of Lecture Notes in Computer Science. Springer Berlin Heidelberg, Madrid, Spain, pp. 847-856.

Minogue, J., Jones, M. G., 2006. Haptics in education: exploring an untapped sensory modality. Review of Educational Research 76 (3), 317-348.

Minogue, J., Jones, M. G., Broadwell, B., Oppewall, T., 2006. The impact of haptic augmentation on middle school students' conceptions of the animal cell. Virtual Reality 10 (3), 293-305.

Mohand Ousaid, A., Millet, G., Régnier, S., Haliyo, S., Hayward, V., 2011. Haptic interface transparency achieved through viscous coupling. The International Journal of Robotics Research, In press.

Moreno, R., Mayer, R. E., Jun. 1999. Cognitive principles of multimedia learning: The role of modality and contiguity. Journal of Educational Psychology 91 (2), 358-368.

Niebert, K., Marsch, S., Treagust, D. F., 2012. Understanding needs embodiment: A theory-guided reanalysis of the role of metaphors and analogies in understanding science. Science Education 96 (5), 849-877.

Okamura, A. M., Richard, C., Cutosky, M. R., Jul. 2002. Feeling is believing: Using a force-feedback joystick to teach dynamic systems. Journal of Engineering Education 91 (3), 345-349.

Papert, S., 1980. Mindstorms: children, computers, and powerful ideas. Basic Books, New York, NY, USA. 
Park, J., Kim, K., Tan, H., Reifenberger, R., Bertoline, G., Hoberman, T., Bennett, D., Mar. 2010. An initial study of visuohaptic simulation of point-charge interactions. In: Proceedings of the IEEE Haptics Symposium 2010. Waltham, MA, USA, pp. 425-430.

Persson, P. B., Cooper, M. D., Tibell, L. A. E., Ainsworth, S., Ynnerman, A., Jonsson, B.-H., Mar. 2007. Designing and evaluating a haptic system for biomolecular education. In: Proceedings of the IEEE Virtual Reality Conference. Charlotte, NC, USA, pp. 171-178.

Piaget, J., 1964. Part i: Cognitive development in children: Piaget development and learning. Journal of Research in Science Teaching 2 (3), 176-186.

Planinšič, G., Kovač, J., 2008. Nano goes to school: a teaching model of the atomic force microscope. Physics Education 43 (1), 37-45.

Podolefsky, N. S., Finkelstein, N. D., Jul. 2006. Use of analogy in learning physics: The role of representations. Phys. Rev. ST Phys. Educ. Res. 2 (2), 020101.

Reiner, M., Apr. 1999. Conceptual construction of fields through tactile interface. Interactive Learning Environments 7 (1), 31-55.

Reiner, M., 2008. Seeing through touch: The role of haptic information in visualization. In: Visualization: Theory and Practice in Science Education. Models and Modeling in Science Education. Springer, pp. 73-84.

Roco, M. C., 2003. Converging science and technology at the nanoscale: opportunities for education and training. Nature Biotechnology 21, 1247-1249.

Sandifer, C., 2004. Spontaneous student-generated analogies. AIP Conference Proceedings 720 (1), 93-96.

Sausse-Lhernould, M., Delchambre, A., Régnier, S., Lambert, P., May 2007. Electrostatic forces in micromanipulations: review of analytical models and simulations including roughness. Applied Surface Science 253 (14), 6203-6210.

Schnotz, W., Bannert, M., 2003. Construction and interference in learning from multiple representation. Learning and Instruction 13 (2), 141-156, external and Internal Representations in Multimedia Learning.

Sitti, M., Mar. 2007. Microscale and nanoscale robotics systems [grand challenges of robotics]. IEEE Robotics and Automation Magazine 14 (1), 53-60.

Sweller, J., 1994. Cognitive load theory, learning difficulty and instructional design. Learning and Instruction 4, 295-312.

Sweller, J., Merrienboer, J. J. G. V., Paas, F. G. W. C., 1998. Cognitive architecture and instructional design. Educational Psychology Review 10 (3), 251-296. 
Tretter, T. R., Jones, M. G., Andre, T., Negishi, A., Minogue, J., 2006a. Conceptual boundaries and distances: Students' and experts' concepts of the scale of scientific phenomena. Journal of Research in Science Teaching 43 (3), 282-319.

Tretter, T. R., Jones, M. G., Minogue, J., 2006b. Accuracy of scale conceptions in science: Mental maneuverings across many orders of spatial magnitude. Journal of Research in Science Teaching 43 (10), 1061-1085.

Trey, L., Khan, S., Sep. 2008. How science students can learn about unobservable phenomena using computer-based analogies. Computers \& Education 51, 519-529.

Tversky, B., Morrison, J. B., Bétrancourt, M., Oct. 2002. Animation: can it facilitate? International Journal of Human-Computer Studies 57 (4), 247-262.

Vogl, W., Ma, B. K.-L., Sitti, M., Jul. 2006. Augmented reality user interface for an atomic force microscope-based nanorobotic system. IEEE Transactions on Nanotechnology 5 (4), 397-406.

Vygotsky, 1978. Mind in society: The development of higher psychological processes. Cambridge, MA: Harvard University Press.

Winn, W., 2003. Learning in artificial environments: Embodiment, embeddedness and dynamic adaptation. Technology Instruction, Cognition and Learning 1 (1), 87-114.

Xie, H., Régnier, S., 2012. High-efficiency automated nanomanipulation with parallel imaging/manipulation force microscopy. IEEE Transactions on Nanotechnology 11 (1), 21-33.

Young, J., Stolfi, C., Tan, H., Chevrier, J., Dick, B., Bertoline, G., june 2011. Learning force concepts using visual trajectory and haptic force information at the elementary school level. In: Proceedings of the IEEE World Haptics Conference (WHC). Istanbul, Turkey, pp. 391-396. 